\title{
Lesión de la rama motora profunda o descendente, o lesión de tipo 3 del nervio interóseo posterior
}

\author{
S. M. Medina Macías ${ }^{(1)}$, J. Ojeda Castellano ${ }^{(2)}$, M. Santana Vélez ${ }^{(3)}$, \\ A. Rivero Alvarado ${ }^{(3)}$ \\ ${ }^{(1)}$ Responsable de la UNIDAD DE Miembro Superior de FREMAP (VECINDARIO) EN LA PROVINCIA DE \\ GRAN CANARIA

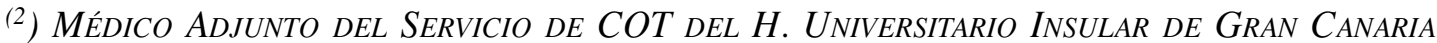 \\ (3) DUE. MIEMBRo de LA UNIDAD DE MIEMbRo SUPERIor dE FREMAP (VECINDARIO) \\ Correspondencia: \\ Dra Sonia Mª Medina Macías \\ Avda. de Canaria 11 \\ 35110, Santa Lucía (Gran Canaria) \\ Tel.: 679192469 \\ e-mail: sonia_medina@fremap.es
}

La parálisis del nervio interóseo posterior (NIP), traumática o no, es poco frecuente. La mayoría de las traumáticas son por complicación de una fractura-luxación de Monteggia y no por una laceración del nervio.

Según las características clínicas y anatómicas, se puede clasificar en tres tipos (afectación del NIP, de la rama recurrente o motora superficial, de la rama descendente o motora profunda).

No se debe retrasar la exploración quirúrgica del nervio más de 3 meses. La reparación de las ramas terminales en el antebrazo es difícil por la dificultad para encontrarlas y por su pequeño calibre. Puede estar indicada la transposición tendinosa con buenos resultados funcionales.

Palabras clave: Nervio interóseo posterior, Nervio motor, Ramas terminales.
The paralysis of the posterior interosseous nerve (PIN), traumatic or no, is little frequent. Most of the traumatic ones they are by complication of a fracture luxación of Monteggia and not by a laceration of the nerve.

According to the clinical and anatomical characteristics, it is possible to be classified in three types (affectation of the NIP, the recurrent or superficial motor branch, the descendent or deep motor branch).

The surgical exploration of the nerve is not due to delay more than 3 months. The repair of the terminal branches in the forearm is difficult by the difficulty to find them and its small caliber. It can be indicated the tendon transfer with good functional results.

Key words: Posterior interosseus nerve, Motor nerve, Terminal branches. 


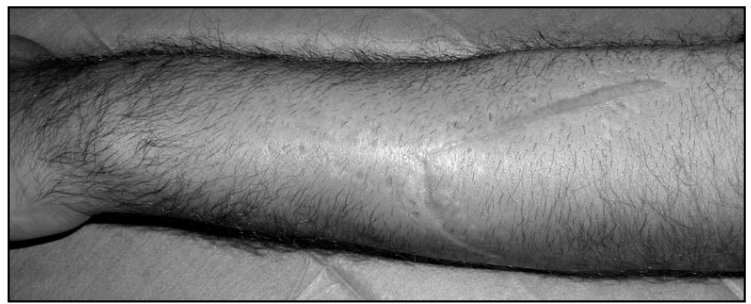

Figura 1: Lesión en la cara posterior del antebrazo izquierdo.

\section{INTRODUCCIÓN}

Entre los síndromes canaliculares y lesiones de los nervios periféricos del miembro superior, la afectación del interóseo posterior (NIP) es poco frecuente, siendo causada en la mayoría de las ocasiones por estructuras anatómicas compresivas, como: el complejo aponeurótico cápsulo-tendinoso de la parte anterior de la articulación humerorradial y la cabeza del radio; vasos recurrentes radiales hipertrofiados por el esfuerzo muscular o arcadas vasculares formadas por la arteria recurrente radial y sus ramas; el borde fibroso o arcada formada por la cabeza medial del extensor carpi radialis brevis; arcada de Fröshe o reborde fibroso proximal del fascículo superficial del supinator, que es membranosa en la mayoría de los casos, pero que también se puede presentar de forma tendinosa; o la presencia de bandas fibrosas entre el fascículo superficial y profundo del supinator ${ }^{1-3}$. La parálisis traumática del NIP es todavía menos frecuente, está relacionada casi siempre con fracturas-luxaciones de Monteggia y, más raramente, con contusiones y laceraciones de diverso tipo a la altura del húmero distal, codo y antebrazo $^{4-5}$.

\section{CASO CLÍNICO}

Se presenta el caso de un varón de 35 años, diestro, que acudió a nuestra consulta 2 meses después de sufrir una lesión por arma blanca en el antebrazo izquierdo (Figura 1). El estudio electromiográfico (EMG) informaba de una "neuropatía axonal grave" del NIP. La exploración clínica puso de relieve un déficit para la extensión y abducción del pulgar, para la extensión de las articulaciones metacarpofalángi-

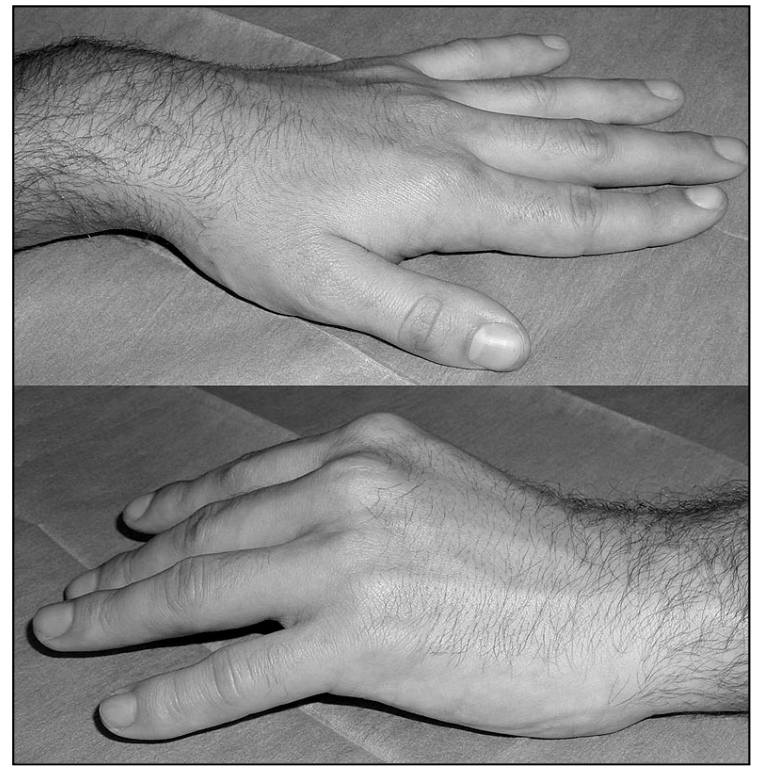

Figura 2: Déficit de extensión y abducción del pulgar; déficit de extensión de la MCF y de la muñeca.

cas (MCF) de los dedos largos y para la dorsiflexión de la muñeca, que realizaba con inclinación radial (Figura 2). Paralelamente se apreciaba pérdida de fuerza y amiotrofia antebraquial. El conjunto de observaciones hizo sospechar una parálisis baja del nervio radial. Por este motivo se indicó la exploración quirúrgica, que tuvo lugar transcurridos 2,5 meses de la lesión. En el acto operatorio se encontró fibrosis acentuada englobando la sutura muscular, realizada durante la urgencia, y las ramas nerviosas. Con esta perspectiva se practicó exoneurolisis del NIP, hallando íntegro el tronco principal y su rama terminal recurrente, mientras que la rama terminal descendente presentaba un neuroma en continuidad, que no fue reparado (Figura 3).

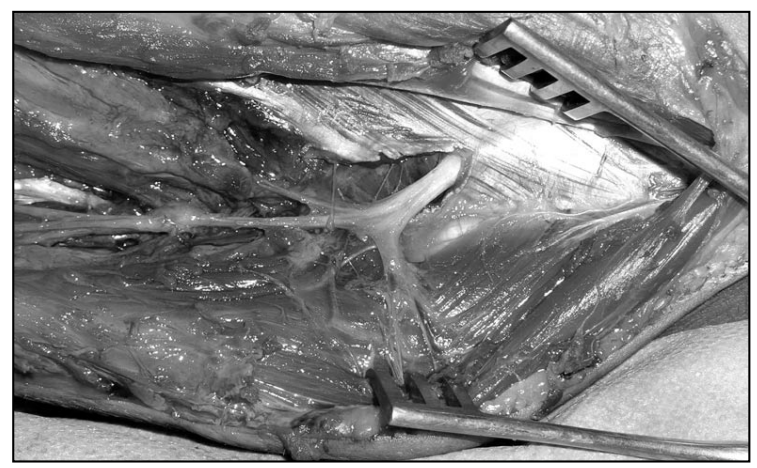

Figura 3: Imagen intraoperatoria: lesión de la rama profunda, motora o descendente. 


\section{Tabla I - VALORAción DE LOS RESULTAdOS EN LA LESIÓN DEL NIP SEGÚN LA ESCALA DE YOUNG ET AL. 5}

\begin{tabular}{|ll|}
\hline Excelente & $\begin{array}{l}\text { grado de fuerza muscular } 5, \text { no pérdida de extensión } \mathrm{MCF} \text {, no desviación radial de } \\
\text { la muñeca cuando se extiende activamente }\end{array}$ \\
\hline Bueno & $\begin{array}{l}\text { grado de fuerza muscular } 4, \text { pérdida de extensión de las } \mathrm{MCF}<15^{\circ} \text {, desviación } \\
\text { radial de la muñeca }<10^{\circ}\end{array}$ \\
\hline Malo & $\begin{array}{l}\text { grado fuerza muscular } 3, \text { pérdida de extensión de las } \mathrm{MCF}>15^{\circ} \text {, desviación ra- } \\
\text { dial de la muñeca }>10^{\circ}\end{array}$ \\
\hline Pobre & sin cambios \\
\hline
\end{tabular}

\section{DISCUSIÓN}

La lesión encontrada durante la intervención corresponde al tipo 3 según la clasificación de Suematsu et al. ${ }^{6}$, y de Hirachi et al. ${ }^{4}$, que evolucionó favorablemente con la exoneurolisis.

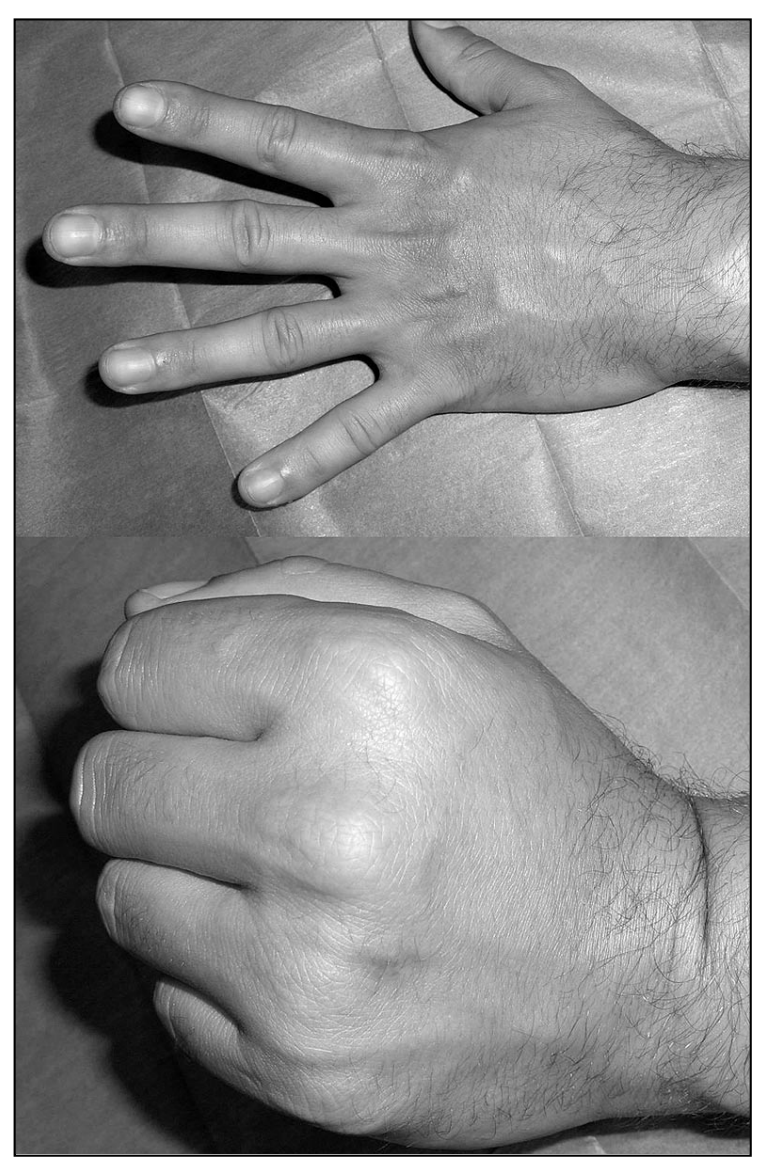

Figura 4: Resultado, a los 12 meses: déficit de extensión de MCF < 15; déficit de extensión y abducción del pulgar; extensión de muñeca con inclinación radial $<10^{\circ}$.
Aunque no procedimos a la reparación de la lesión de la rama terminal descendente, los resultados funcionales tras la cirugía no exigieron maniobra paliativa alguna mediante transferencias tenomusculares. El paciente siguió tratamiento rehabilitador y medicación con pregabalina. A los 3 meses de la cirugía fue dado de alta, reincorporándose a su trabajo como vigilante y efectuando con normalidad su actividad deportiva habitual. Este resultado se contempla como "bueno" según la escala utilizada en la serie publicada por Young et al. ${ }^{5}$ (Tabla 1), con normalización del trofismo muscular, recuperación prácticamente completa de la extensión de las MCF de los dedos largos y de la dorsiflexión de la muñeca, que mantuvo una inclinación radial menor de $10^{\circ}$ (Figura 4). La recuperación de la abducción y extensión del pulgar fue incompleta (Figura 4), existiendo también un déficit de recuperación de fuerza de la mano (grado 4 MRC) valorado según el sistema de la British Medical Research Council ${ }^{5,7}$.

En general, la parálisis del NIP no es frecuente, existiendo pocas series publicadas de pacientes tratados quirúrgicamente ${ }^{5}$. La parálisis de origen traumático es más rara todavía, y la mayoría de las veces se presenta como complicación de una fractura-luxación de Monteggia ${ }^{1,4-5}$.

El nervio radial emerge del cordón posterior del plexo braquial, que toma sus fibras de las raíces C5 a T1. Llega al antebrazo por la región lateral, entre los músculos braquial y braquiorradialis, y en esta zona proximal al codo, 
da ramas musculares para los músculos braquiorradialis, extensor carpis radialis longus, ancóneo y extensor carpis radialis brevis ${ }^{8}$, aunque hay estudios anatómicos que determinan que en un $26 \%$ de ocasiones la inervación del extensor carpis radialis brevis depende también del NIP ${ }^{3}$. Al llegar al canal bicipital externo se divide en sus dos ramas terminales: la rama superficial sensitiva, que sigue en profundidad el trayecto del braquiorradialis, y la rama profunda, motora o NIP, que pasa entre los dos fascículos del supinator, y por la arcada de Fröshe, que constituye un refuerzo fibroso de su fascículo superficial ${ }^{8}$.

La rama motora se divide cerca de su salida bajo el extremo distal del supinator en sus dos ramas terminales ${ }^{6}$ : a) una recurrente o rama motora superficial, que inerva al extensor digitorum communis, al extensor digiti minimi $\mathrm{y}$ al extensor carpi ulnaris y, b) otra descendente o rama motora profunda, que inerva al abductor pollicis longus, al extensor pollicis brevis, al extensor pollicis longus y al extensor indicis propius.

La parálisis completa del NIP, se manifiesta por impotencia para la extensión de la muñeca, con un componente de desviación radial dado el predominio de los extensores radiales ante la parálisis del extensor carpi ulnaris, o también al predominio del extensor carpis radialis longus sobre el brevis, cuando este último depende del NIP, como hemos mencionado $^{3}$; pérdida de extensión de las articulaciones MCF de los dedos largos y del pulgar, debilidad para la abducción del pulgar ${ }^{4,5}$.

Según las características clínicas y anatómicas, las parálisis del NIP se pueden clasificar en tres tipos ${ }^{4,6}$ :

- Tipo 1: parálisis completa por compresión del tronco principal o compresión de ambas ramas, proximalmente o bajo el supinator.

- Tipo 2: pérdida de extensión de los dedos tercero, cuarto y quinto (funcionales primero y segundo), por compresión aislada de la rama recurrente a su salida o distalmente al supinator.

- Tipo 3: pérdida de extensión del primer y segundo dedos (funcionales el resto de los dedos), así como de la abducción del pulgar, debida a la compresión aislada de la rama des- cendente también a su salida o distalmente al supinator. Este es el tipo de lesión hallada en el caso clínico que ahora presentamos.

Entre las pruebas complementarias utilizadas para el diagnóstico, los resultados EMG no siempre coinciden con los hallazgos clínicos o quirúrgicos, y la identificación de una lesión completa o incompleta basada en este estudio puede conducir a error ${ }^{5}$. En este sentido, la mayoría de los autores establecen que, independientemente del origen de la lesión, no se debe posponer la exploración quirúrgica del nervio más allá de tres meses, pudiendo indicarse incluso a las seis semanas si no hay evidencia clínica ni EMG de recuperación ${ }^{4,5}$. En general, sí a los tres meses existe recuperación clínica del paciente concluiremos que la lesión se trata de una neuropraxia, cuyo tratamiento debe ser conservador. Por lo contrario, si no hay evidencia de recuperación clínica o EMG, cabe sospechar lesión por laceración nerviosa, quedando formalmente indicada la revisión quirúrgica antes de que se produzca la atrofia muscular ${ }^{5}$.

En las escasas publicaciones existentes sobre el tratamiento de las lesiones traumáticas del NIP, se acepta la dificultad para encontrar las ramas terminales y proceder a su reparación mediante la resección de los extremos del nervio hasta el tejido fascicular sano, con posterior neurorrafia o mediante injerto nervioso. En algunos casos de lesión irreparable puede estar indicada la cirugía paliativa mediante transposiciones tendinosas, con buenos resultados funcionales $^{4,5}$.

En la serie publicada por Young et al., la recuperación de los pacientes se produjo en 2 años y 4 meses, con un rango de 1 año y 4 meses a 12 años y 5 meses, del postoperatorio), no habiendo presentado ninguno de ellos un resultado "excelente", según la propia escala del autor". En el caso clínico presentado, el paciente se incorporó a su trabajo y actividad deportiva habitual a los 3 meses de la cirugía, situación que se mantenía al cabo de un año de la intervención. Cabe la posibilidad de que en este resultado haya influido el hecho de que la lesión afectaba la extremidad no dominante, siendo por ello menor la exigencia funcional. 


\section{BIBLIOGRAFÍA}

1. Allieu $\mathrm{Y}$, Chammas M, Roux JL. Síndromes de los canales y desfiladeros (con excepción del canal carpiano). Enciclopedia Médico Quirúrgica (EMC) del Aparato Locomotor. Paris: Editorial Elselvier, 1997: 14-462.

2. Sponseller PD, Engber WD. Double-entrapment radial tunnel syndrome. J Hand Surg Am, 1983; 8: 420-3.

3. Thomas SJ, Yakin DE, Parry BR, Lubahn JD. The anatomical relationship between the posterior interosseous nerve and the su- pinator muscle. $\mathrm{J}$ Hand Surg Am, 2000; 25: 936-41.

4. Hirachi K, Kato H, Minami A, Kasashima T, Kaneda K. Clinical features and management of traumatic posterior interosseous nerve palsy. J Hand Surg Br, 1998; 23: 413-7.

5. Young C, Hudson A, Richards A. Operative treatment of palsy of the posterior interosseous nerve of the forearm. J Bone Joint Surg Am, 1990; 72: 1215-9.

6. Suematsu N, Hirayama T. Posterior interosseous nerve pal- sy. J Hand Surg Br, 1998; 23: 104-6.

7. Millesi H, Meissl G, Berger A. Further experience with interfascicular grafting of the median, ulnar and radial nerves. $J$ Bone and Joint Surg Am, 1976; 56: 209-18.

8. Llusá M, Merí Á, Ruano D. Cintura escapular y miembro superior. Neurología. En: Manual y atlas fotográfico de anatomía del aparato locomotor. Madrid: Editorial Médica Panamericana, 2003: 173-96. 